\title{
Preface : Invited Issue Editor Professor Dachling Pang and the Changing Concepts in Spinal Dysraphism during the Last Two Decades
}

\author{
Chae-Yong Kim, ${ }^{1,2}$ Seung-Ki Kim ${ }^{1}$ \\ Editor of the 'Pediatric Issue,', Journal of Korean Neurosurgical, Seoul, Korea \\ Chair, ${ }^{2}$ Publication Committee, Korean Society of Pediatric Neurosurgery, Seoul, Korea
}

Since 2015, the Korean Society for Pediatric Neurosurgery (KSPN) has published an official journal known as the Pediatric Issue as a supplement to the Journal of Korean Neurosurgical Society (JKNS), which is the official journal of the Korean Neurosurgical Society. The Pediatric Issue is published in May each year, particularly to honor Children's Day on May 5 and the annual meeting of the KSPN that is held in May. One of the figures presented in the Pediatric Issue is selected to be on the cover of the May issue of the journal.

The editorial board of the JKNS selected the six most influential articles from the JKNS publications between 2016 and 2018. These articles captured the academic interests of international scientific communities. Two of the six most influential articles came from editions of the Pediatric Issue ${ }^{1)}$. One is 'From Exoscope into the Next Generation" ${ }^{\text {'3) }}$, written by Dr. Kenichi Nishiyama of Niigata Medical Center (the first and corresponding author) and edited by Professor Joong-Uhn Choi (invited editor). The other is 'Development and Growth of the Normal Cranial Vault : An Embryologic Review ${ }^{22}$, written by Sung-Won Jin (the first author) and Ki-Bum Sim of Jeju National University Hospital (the corresponding author) and edited by Professor Joon-Ki Kang (invited editor).

The Pediatric Issue has made a significant contribution to the enhancement of the academic level of the JKNS, as well as the field pediatric neurosurgery, by covering a wide range of topics from 2015 to 2019, such as Moyamoya disease (edited by Professor Kyu-Chang Wang and Seung-Ki Kim), craniosynostosis (invited editor : Professor Joon-Ki Kang), the era of neuroendoscopy (invited editor : Professor Joong-Uhn Choi), pediatric malignant brain cancers in the molecular era (invited editor : Professor James T. Rutka), and pediatric epilepsy surgery (invited editor : Professor Byung-Kyu Cho). The topic of the 2020 JKNS : Pediatric Issue, i.e., changing concepts in spinal dysraphism during the last two decades, was organized by the issue's invited editor, Professor Dachling Pang, who is one of the world-famous pioneers in the field of spinal dysraphism (Fig. 1). This is one of two parts that will be published regarding spinal dysraphism. The second part will be published in the 2021 Pediatric Issue 2021 (invited editor : Professor Kyu-Chang Wang).

Professor Dachling Pang graduated with a BSc and the Highest Honours Medal in physiology and biophysics at Dal-

- Received : March 4, 2020 •Revised : March 16, 2020 •Accepted : March 31, 2020

- Address for reprints : Seung-Ki Kim

Division of Pediatric Neurosurgery, Seoul National University Children's Hospital, Seoul National University College of Medicine, 101 Daehak-ro, Jongno-gu, Seoul 03080, Korea

Tel : +82-2-2072-2350, Fax : +82-2-744-8459, E-mail : nsthomas@snu.ac.kr, ORCID : https://orcid.org/0000-0002-0039-0083

This is an Open Access article distributed under the terms of the Creative Commons Attribution Non-Commercial License (http://creativecommons.org/licenses/by-nc/4.0) which permits unrestricted non-commercial use, distribution, and reproduction in any medium, provided the original work is properly cited. 


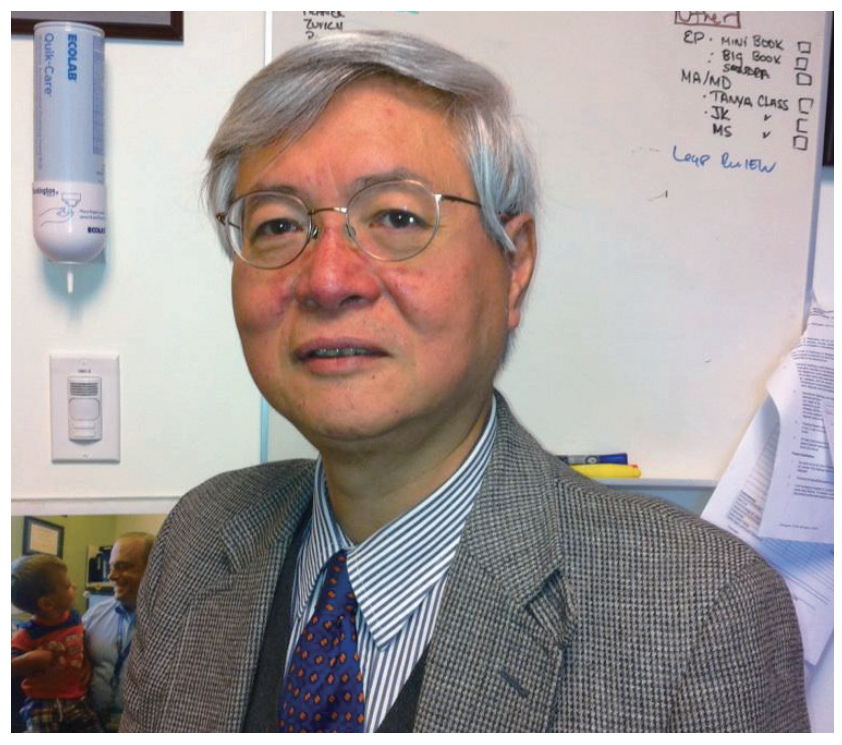

Fig. 1. Invited Issue Editor, Professor Dachling Pang.

housie University, Halifax, Nova Scotia, Canada, before entering medical school at the University of Toronto, where he obtained his MD in 1972. He completed his residencies in general surgery and neurosurgery in 1977 at Toronto and then a fellowship in pediatric neurosurgery at the Hospital for Sick Children in Toronto in 1978.

He holds specialist certificates in the American Board of Neurological Surgery, the American Board of Pediatric Neurological Surgery, the Royal College of Surgeons, and the Royal College of Surgeons of Canada. He has been elected a fellow by the Royal Colleges of Surgeons of Canada and the United Kingdom and the American College of Surgeons.

He joined the faculty of the University of Pittsburgh as an assistant professor of pediatric neurosurgery in 1979; he then became the chief of pediatric neurosurgery at Children's Hospital of Pittsburgh and a full professor at the University of Pittsburgh in 1987. He served in this capacity until 1993, when he moved to be a professor and the chief of pediatric neurosurgery at the University of California, Davis. In 2003, he also took on the position of chief of the Regional Center of Pediatric Neurosurgery of Northern California Kaiser Foundation Hospitals. Since 2017, he has served as an honorary consultant pediatric neurosurgeon at the Great Ormond Street Hospital for Children in London, United Kingdom, mainly as a teacher and mentor for other pediatric neurosurgery consultants and fellows.

Widely acclaimed internationally for his pioneering work on disorders of the pediatric spine, especially in the field of spinal dysraphism and other congenital malformations, Professor Pang was conferred an adjunct professorship from Nanjing Medical University, China, a honorary professorship from Sun Yat-Sen University, China, and a visiting professorship from the University of Cape Town, South Africa. He is also an honorary member of the Neurosurgical Society of Hong Kong. He has been an invited speaker on over 370 occasions throughout many countries and has also been guest surgeon in many places, including Hong Kong, Singapore, India, Beijing, Nanjing, Guangzhou in China, Taiwan, South Africa, and Thailand.

With research interests encompassing neuroembryology, pediatric spinal injuries, spinal cord congenital malformations, embryology and bony malformations of the craniovertebral junction, intraoperative neurophysiology monitoring, and neuroendocrinology, he has been the principle recipient of a National Institute of Health RO1 Grant on intraventricular hemorrhage in a canine model and a Center Project Grant from the University of Pittsburgh on the induction of precocious puberty using a noninvasive balloon in the third ventricle in a primate model. His published list includes over 200 refereed articles and chapters, many of which are landmark publications. He has edited two books on pediatric spine and spinal cord diseases, in addition to currently serving as co-editor-in-chief for a textbook of pediatric neurosurgery project.

He now lives full time in London with his wife, Lynne.

Dr. Pang was invited to be the editor of the 2020 Pediatric Issue: Changing Concepts in Spinal Dysraphism during the Last Two Decades due to his remarkable contribution to the field.

Recent advances in the understanding of the anatomical pathology and pathoembryology of congenital disorders of the nervous system have led to changes in the classification and management of spinal dysraphism. For example, limited dorsal myeloschisis has been summarized as a distinctive clinicopathological entity, and complete untethering, radical fat removal, pial reconstruction and spacious dural reconstruction are emerging as a new treatment strategies for lumbosacral lipomatous malformation. The articles of this Pediatric Issue will undoubtedly provide readers with current concepts and future directions regarding pediatric spinal dysraphism. Ten articles were contributed by leading researchers in this field from Korea, the United Kingdom, and Germany. 
Professor Pang actively recruited world-class scholars to participate in the writing of this Pediatric Issue. Dr. Eibach of Germany provided a novel concept on the junctional neurulation defect. Current and future perspectives were summarized by Professor Pang at the end of the edition. Furthermore, he also recruited outstanding Korean scholars, Dr. Kyu-Chang Wang, Dr. Ji Yeoun Lee, Dr. Kwan Jin Park, and Dr. Kyung Hyun Kim, who are specialists in this field.

We deeply appreciate all of the experts from throughout the world and Korea for their active participation in the $2020 \mathrm{Pe}$ diatric Issue. Additionally, we would like to express our sincere gratitude to Professor Pang for his enthusiasm and dedication as the invited editor.

\section{CONFLICTS OF INTEREST}

The authors have no financial conflicts of interest.

\section{AUTHOR CONTRIBUTIONS}

\author{
Conceptualization : CYK, SKK \\ Data curation : CYK \\ Project administration : SKK \\ Visualization : CYK, SKK \\ Writing - original draft : CYK, SKK \\ Writing - review \& editing : CYK, SKK
}

\section{ORCID}

Chae-Yong Kim https://orcid.org/0000-0001-9773-5553

Seung-Ki Kim https://orcid.org/0000-0002-0039-0083

\section{References}

1. Im SB, Chung M, Kim BT : The influential articles and title words in the Journal of Korean Neurological Society Publications between the years 2016 to 2018. J Korean Neurosurg Soc 63 : 1-3, 2020

2. Jin SW, Sim KB, Kim SD : Development and growth of the normal cranial vault: an embryologic review. J Korean Neurosurg Soc 59 : 192-196, 2016

3. Nishiyama K : From exoscope into the next generation. J Korean Neurosurg Soc 60 : 289-293, 2017 